\title{
Apoptotic Process Induced by Oxaliplatin in Rat Hippocampus Causes Memory Impairment
}

\author{
Enrica Bianchi ${ }^{1}$, Lorenzo Di Cesare Mannelli ${ }^{\mathbf{2}}$, Laura Micheli ${ }^{2}$, Mersedez Farzad ${ }^{\mathbf{3}}$, Margherita Aglianò ${ }^{\mathbf{1}}$ and Carla Ghelardini ${ }^{2}$ \\ ${ }^{1}$ Department of Medicine, Surgery and Neuroscience, University of Siena, Siena, Italy, ${ }^{2}$ Department of Neuroscience, Psychology, Drug Research \\ and Child Health, Neurofarba, Pharmacology and Toxicology Unit, University of Florence, Florence, Italy and ${ }^{3}$ Oncology Unit, Healthcare \\ Institution Tuscan Southeast, Siena, Italy
}

(Received 2 February 2016; Accepted 2 June 2016)

\begin{abstract}
Aspects of memory involved in cognitive mechanisms were investigated in rat after oxaliplatin (OX) chemotherapy using animal behavioural assessment of passive avoidance and social learning paradigms, which are both hippocampus-sensitive. Rodents, previously subjected to 2-week OX treatment, showed passive avoidance and social learning impairment and apoptotic processes in the hippocampus. Apoptosis rate significantly increased in cultured hippocampal cells exposed to OX at increasing doses, and this effect was dose-dependent. Ex vivo experiments showed that cell damage and apoptosis were blocked in the hippocampus from OX rats cotreated with copper sulphate (CS) which precludes OX transport inside the cell. In vivo, passive avoidance and social learning impairment could not be observed in OX rats co-administered with CS. Thus, a site of action of OX treatment on memory impairment appears to be located at the hippocampus. These findings strongly support that cellular damage induced by $\mathrm{OX}$ in rodent hippocampus underlies the weakening of some memory functions.
\end{abstract}

Several studies have reported that chemotherapy could produce cognitive dysfunction. Even if mild-to-moderate cognitive impairment usually occurs, it can break down quality of life, work performance and daily activities [1]. Some studies described the disorder as temporary; others reported difficulties in cognition that could last up to 20 years after treatment termination $[2,3]$.

Oxaliplatin (oxalato(transl-1,2-diaminocyclohexane) (OX) is an alkylating agent able to bind to the DNA strands causing alterations that trigger apoptosis [4]. It has been found to penetrate the BBB and accumulate in both cerebrospinal and extracellular fluid in the brain of non-human primate [5]. Previous studies demonstrated that cognitive functions were modified in behavioural tests performed in animals submitted to OX treatment up to 11 months after treatment completion [6]. However, it is presently unknown how OX might cause cognitive impairments. We focused on two aspects of memory involved in cognitive mechanisms such as passive avoidance and social recognition. Hippocampus is a key structure involved in the induction of these forms of memory [7-10]. Therefore, we investigated in rodents how OX might cause impairment on social memory and passive avoidance with regard to cellular alteration induced in the hippocampus by the chemotherapeutic agent.

\section{Materials and Methods}

Animal care. All animal manipulations were performed according to the European community guidelines for animal care (DL 116/92,

Author for correspondence: Enrica Bianchi, Department of Medicine, Surgery and Neuroscience, Via A. Moro 6- 53100 Siena, Italy (e-mail enrica.bianchi@unisi.it).
Application of the European Communities Council Directive of 24 November 1986, 86/609/EEC; NIH publication no. 85-23, revised 1996; University of Florence assurance number: A5278-01). All studies involving animals were in accordance with the ARRIVE guidelines for experiments involving animals [11]. A total of 144 animals were used in these experiments. Adult (12-15 weeks) male rats were purchased from Morini (Morini, Italy). Animals were given food and water ad libitum and maintained under a reversed light cycle (10:00 hr light off, 22:00 hr light on). Experiments were always performed during the nocturnal portion of the reversed light cycle. To minimize the number of animals used, sample size as related to statistical significance was considered as described in Feinstein [12].

Animal treatment design. Animals were randomly attributed to the following groups: (i) intraperitoneal (i.p.) vehicle control; (ii) intrahippocampus (i.h.) saline control; (iii) $\mathrm{OX}$; (iv) $\mathrm{OX}+\mathrm{CS}$; and (v) CS. The OX group was administered OX dissolved in $5 \%$ glucose solution (vehicle) (Braun, Milano, Italy) once a day at a dose of $2.4 \mathrm{mg} / \mathrm{kg}$ i.p for five consecutive days every week for 2 weeks according a previous established protocol [13]. The vehicle control group was administered 5\% glucose solution once a day i.p. for five consecutive days every week for 2 weeks. The OX + CS group was co-administered i.p. OX and bilaterally i.h.-injected copper sulphate (CS) dissolved in saline at a dose of $0.5 \mu \mathrm{g} / \mathrm{rat}$. The CS group was bilaterally i.h.-administered CS alone dissolved in saline. The 10- $\mu \mathrm{g}$ cumulative CS dose was established to not induce toxicity in the animals [14] and to be effective in reverting the effects of OX in the passive avoidance and in social learning test.

Intrahippocampal administration. Animals were anaesthetized by i.p. administration of ketamine hydrochloride $(60 \mathrm{mg} / \mathrm{kg}$ ) and xylazine $(4 \mathrm{mg} / \mathrm{kg})$ and then positioned in a stereotaxic apparatus. Body temperature was maintained with the aid of a heating pad. The depth of anaesthesia was determined by measurement of pedal reflex. A stainless steel cannula (22 gages) was implanted in the CA1 of the dorsal hippocampus at $-2.6 \mathrm{~mm}$ anterocaudal, $\pm 2 \mathrm{~mm}$ lateral (with respect to the bregma) and $3.3 \mathrm{~mm}$ vertical coordinates according to 
the atlas of Paxinos and Watson [15]. After surgery, the rats were allowed to recover for 7 days. The drug solutions were injected into the hippocampus by microinjection unit attached to a $2-\mu 1$ Hamilton microsyringe via polyethylene tubing. The left and the right hippocampus were injected with $0.5 \mu \mathrm{l}$ of solution on each side. Administration was controlled by an infusion pump programmed to deliver a volume at rate of $0.1 \mu \mathrm{l}$ over a period of $30 \mathrm{sec}$.

\section{Behavioural tests.}

Passive avoidance test. Passive avoidance learning and retention were assessed using a step-through paradigm modelled according to Jarvik and Koop [16]. Throughout the acquisition/conditioning phase (training), the rat is placed in a lighted compartment. When the animal crosses to the dark compartment, it receives a mild foot shock. During the test phase (retention), the animal is again placed in the lighted compartment. The differences between the second and the first entry latency into the dark compartment were calculated. The increase in avoidance latency indicated an improved avoidance learning behaviour.

Social memory assessment. Social memory test is based on the propensity of an adult rodent to inspect an unknown juvenile rodent [17]. A never-before-met juvenile rat is placed into the home cage of a test adult animal for $5 \mathrm{~min}$. and then removed (1st exposure). After $24 \mathrm{hr}$, the same juvenile rat is placed back in the home cage together with a novel, never-before-met juvenile rat for $5 \mathrm{~min}$. (2nd exposure). All test trials are videotaped and subsequently analysed for total body, anogenital and perioral investigation and grooming behaviour. In the second session, the adult rat has a free choice between the first, already-investigated, juvenile rat and a novel unfamiliar rat. Usually, an animal with intact social memory recalls its previous contact with the before-met rat, and it tends to spend more time with the newly encountered rat.

Olfaction test. Foraging test. Food was set aside from individually housed rats for 16-24 hr before testing [18]. Then, small pieces of chocolate were made accessible to rats for $12 \mathrm{hr}$, and chow was given to rats when chocolate was fully eaten. Two days later, food was set aside away for 16-24 hr. Then, the rat was transferred from his home cage to a holding cage. Successively, a small piece of chocolate was set on the bedding of the cage and the rat returned to his home cage. The latency to detect chocolate chip was measured. The procedure was repeated three more times locating small pieces of chocolate in different positions beneath $2-3 \mathrm{~cm}$ of levelled bedding.

Olfactory habituation test. A Small perforate tube was filled with cotton, added with lemon natural extract $(10 \mu \mathrm{l})$ and placed in the cage of each rat for $1 \mathrm{~min}$. [19]. The procedure was repeated four times at $10-\mathrm{min}$. intervals. In a 10-min. later trial, vanilla natural extract $(10 \mu \mathrm{l})$ was added to lemon in the tube which was set in the rat cage. Olfactory investigation (nasal contact with the tube) was recorded.

Motor co-ordination and exploratory ability tests. Groups of rats treated with all the drugs used in the above experiments were submitted to rotarod [20] and hole-board [20] behavioural tests. In the rotarod test, the rat was placed on a horizontal rotating rod; the animal must walk forward to remain upright and not fall off. The balance time before falling was measured over time. The cut-off time for each measurement was set at $30 \mathrm{sec}$. The hole-board apparatus consists of an enclosed space, the floor of which has sixteen holes in a grid pattern. The rodent, when placed in the apparatus, is free to dip its head through the holes in the floor; the frequency and duration of this behaviour are thought to measure levels of exploratory behaviour.
Oligonucleosomal ELISA. Primary hippocampal cell lines (Life Technologies, Milan, Italy) were cultured according to the manufacturer's instructions. Briefly, cells were cultured on tissue culture flasks in complete neurobasal medium (Thermo Fisher, Waltham, MA, USA) at $37^{\circ} \mathrm{C}$ in air supplemented with $5 \% \mathrm{CO}_{2^{-}}$ humidified conditions. Cells were seeded at a density of $1 \times 10^{4}$ cells/well in $200 \mu \mathrm{l}$, washed three times with neurobasal medium and exposed to $\mathrm{OX}$ at different concentrations for $48 \mathrm{hr}$ at $+37^{\circ} \mathrm{C}$. All experiments were performed in triplicate, a minimum of three times. DNA fragmentation generated during apoptosis in response to $\mathrm{OX}$ treatment was detected using a sandwich enzyme immunoassay system (Cell Death Detection ELISA; Roche, Monza, Italy) that allowed specific determination of mono- and oligonucleosomes in the cytoplasmic fraction of cell lysates. The amount of DNA fragments was determined colorimetrically with the ABTS ELISA Peroxidase Substrate (2,2'-azino-bis(3ethylbenzothiazoline-6-sulphonic acid)) (Life Technologies) using a microplate reader at $405 \mathrm{~nm}$ with a reference filter of $490 \mathrm{~nm}$. Absorbance values were calculated and reported as per cent of control.

\section{Study of apoptosis factors ex vivo.}

Measurement of caspase- 3 and caspase-9 activities. Caspase-3 and caspase- 9 were determined by colorimetric kit (Abcam, Cambridge, UK; MBL, Woburn, MA, USA) following the manufacturer's instructions. All the animals were deeply anaesthetized using thiopental $(50 \mathrm{mg} / \mathrm{kg}$, i.p.), and hippocampus tissues were isolated. Homogenate cells were centrifuged at $450 \times g$ for $10 \mathrm{~min}$., and the supernatant was recentrifuged at $12,000 \times g$ for $10 \mathrm{~min}$. The extracts, each containing $100 \mu \mathrm{g}$ protein, were mixed with $50 \mu \mathrm{l}$ of $2 \times$ reaction buffer/ dithiothreitol (DTT). Next, $5 \mu \mathrm{l}(1 \mathrm{mM})$ of protein substrate (DEVDpNA for caspase-3 and LEHD-pNA for caspase-9) was added to each reaction and incubated at $37^{\circ} \mathrm{C}$ for $3 \mathrm{hr}$. Light emission of pNA was quantified using an ELISA reader (wavelength: 400-405 nm). Absorbance of pNA in the treated as compared to control groups was used as measure of the apoptotic activity. Protein concentration was determined by Protein DC Assay Kit (Bio-Rad, Milan Italy).

Measurement of Bax and Bcl-2 content. ELISA kit containing microtiter plate pre-coated with an antibody specific to Bax and $\mathrm{Bcl}-2$ was used to measure $\mathrm{Bax}$ and $\mathrm{Bcl}-2$ content (Antibodies-online Inc.,

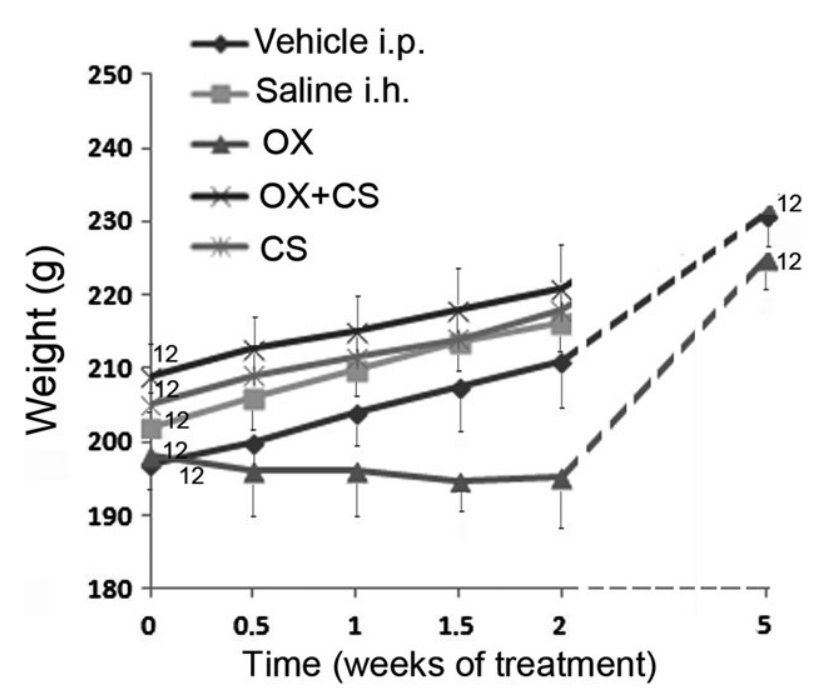

Fig. 1. Time course of rat body-weight in the treatment period. Verti$\mathrm{cal}$ bars represent S.E.M. Reported numbers represent the number of animals used for each experimental group. $F_{4,55}=1.18$, n.s. 
Atlanta, GA, USA). Hippocampal tissue was rinsed with $1 \times$ PBS (phosphate-buffered saline), homogenized in $1 \mathrm{ml}$ of $1 \times$ PBS and stored overnight at $-20^{\circ} \mathrm{C}$. After two freeze-thaw cycles, homogenates were centrifuged for $5 \mathrm{~min}$. at $5000 \times g$ at $2-8^{\circ} \mathrm{C}$ and supernate was immediately assayed. A total of $100 \mu \mathrm{l}$ of standard or unknown sample solution was added to each well with the biotin-conjugated antibody preparation. After $2-\mathrm{hr}$ incubation $\left(37^{\circ} \mathrm{C}\right)$ and washing (three times), $100 \mu \mathrm{l}$ of avidin conjugated to horseradish peroxidase was added to each microplate well and incubated for $30 \mathrm{~min} .\left(37^{\circ} \mathrm{C}\right)$. After washing, $90 \mu \mathrm{l}$ of TMB $\left(3,3^{\prime}, 5,5^{\prime}\right.$-tetramethyl-benzidine) substrate solution was

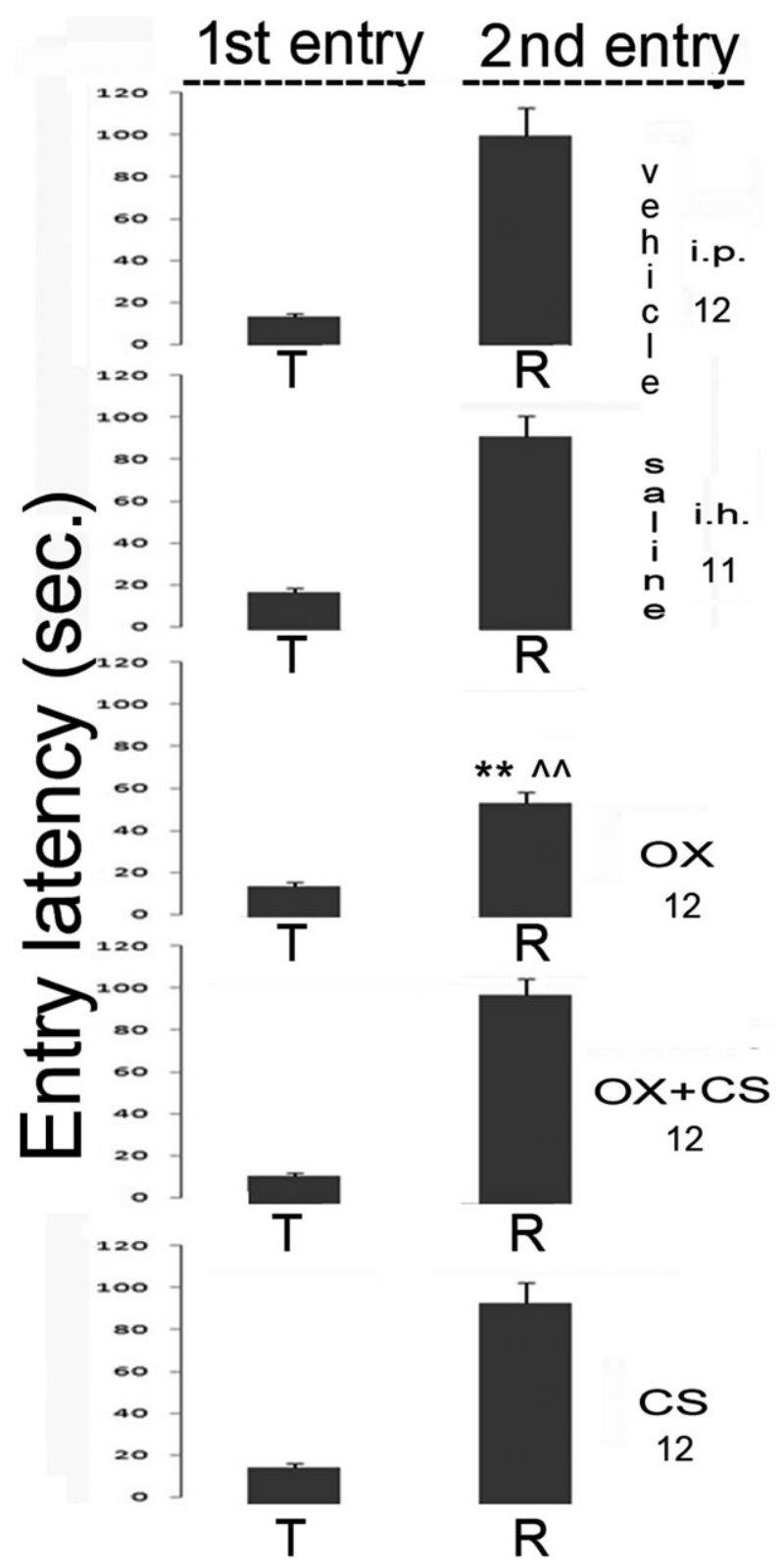

Fig. 2. Entry latency into the dark compartment in the passive avoidance test is shown at the end of OX treatment in the presence or absence of CS. Entry latency values were measured in the training test (T) and after $24 \mathrm{hr}$ in the retention test $(\mathrm{R})$. The numbers shown in the figure represent the number of animals used in each experiment. Vertical lines represent S.E.M. ${ }^{* *} \alpha<0.01$ and ${ }^{\wedge} \wedge \alpha<0.01$ in comparison with corresponding vehicle and saline controls, respectively. $F_{4,54}=5.72, \alpha<0.001$. added to the well and incubated for $15-25 \min .\left(37^{\circ} \mathrm{C}\right)$. Finally, the enzyme-substrate reaction was terminated by the addition of a $50 \mathrm{ml}$ stop solution, and colour change was measured spectrophotometrically reading at $450 \mathrm{~nm}$ wavelength. Protein concentration in unknown samples was determined using standard curve (achieved by standard solution) and expressed as $\mathrm{pg} / \mathrm{ml}$.

Cytosolic cytochrome expression. Mitochondrial fraction from rat hippocampus was obtained using Mitochondria Isolation Kit (Sigma, Milan, Italy). Protein extraction of both the mitochondrial and cytosolic fractions was performed from hippocampal tissue of rats under different treatment conditions. Samples were submitted to Western blot according to the procedure previously described [21]. The primary antibody was at 1:1000 dilution of cytochrome $c$ monoclonal (PharMingen, San Diego, CA, USA).

Histological control. At the conclusion of the behavioural experiments, $1 \%$ Evans blue dye was administered to rats according to

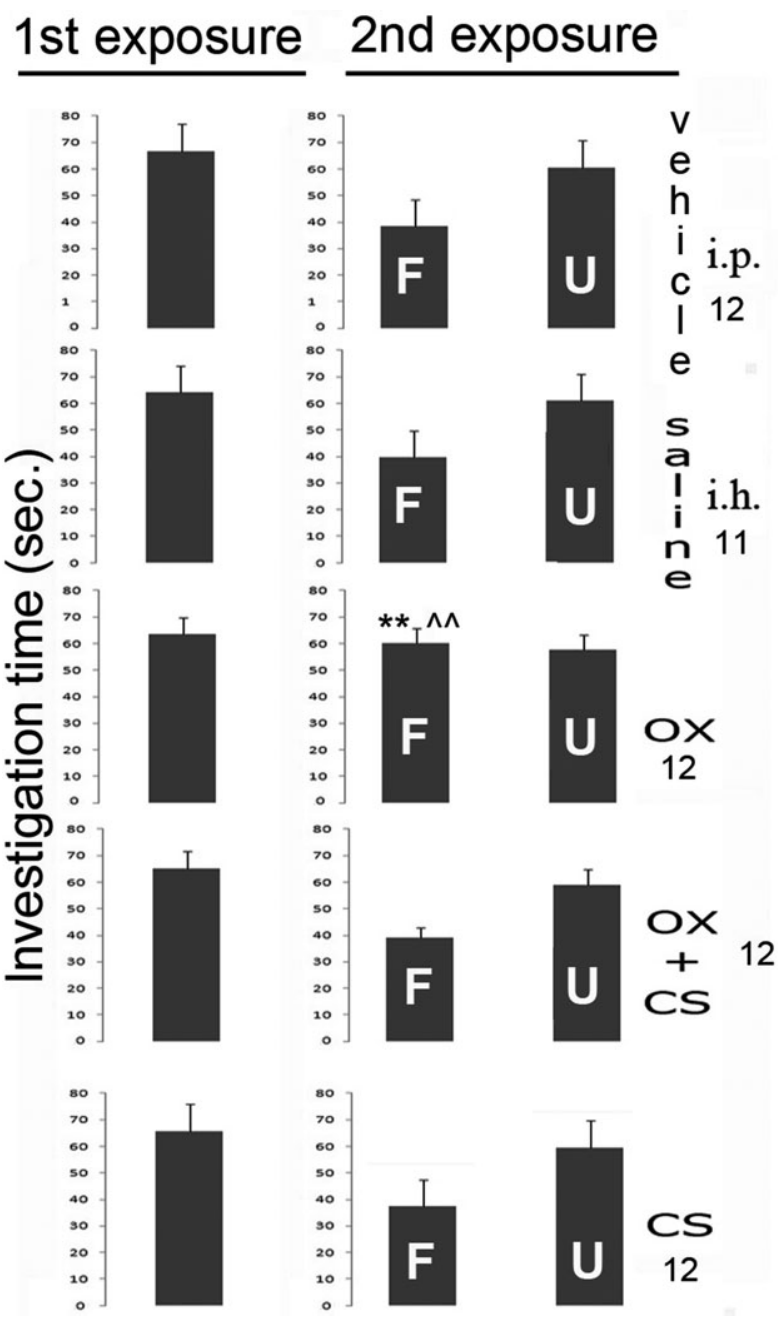

Fig. 3. Investigation times of juvenile by adult rat are shown during the first exposure and on re-exposure after $24 \mathrm{hr}$. Data are shown for familiar (F) and unfamiliar (U) rat after 2-week OX treatment. The same experiment was repeated in OX rats cotreated with CS. The numbers shown in the figure represent the number of animals used in each experiment. Vertical lines represent S.E.M. ${ }^{* *} \alpha<0.01$ and $\wedge^{\wedge} \alpha<0.01$ in comparison with corresponding vehicle and saline controls, respectively. $F_{4,54}=6.12, \alpha<0.001$. 
the microinjection procedure for i.h. administration. A post-mortem histological control of the location of cannula inside the hippocampus was performed on cryostat sections of unfixed brains observed under stereomicroscope (Leica MZ12.5; Leica, Solms, Germany). Data of any rat were excluded from statistical analysis if the cannula tip was outside the CA1 region of the hippocampus or if the region had sustained extensive damage.

Statistics. All experimental results were presented as mean \pm S.E.M. Analysis of variance (ANOVA) followed by Fisher's protected least significant difference procedure for post hoc comparison was used to verify significance between two means of behavioural tests or apoptotic process investigation. Data were analysed with the StatView software for the Macintosh (1992). A significance level $(\alpha)<0.01$ was considered significant.

\section{Results}

Effect of $O X$ on rat weight.

Before initiation of $\mathrm{OX}$ administration, rat groups were weighed in grams, respectively, $195 \pm 8.0$ (vehicle i.p. control), $202.4 \pm 7.3$ (saline i.h. control), $198 \pm 8.1$ (OX), $209 \pm 8.7(\mathrm{OX}+\mathrm{CS})$ and $205 \pm 7.3$ (CS). Statistical comparison between groups showed that the treated and control groups gained weight not significantly differently throughout the 2 weeks of treatment although the weight increase was lower in the OX-treated rats (fig. 1). Weight was not statistically different in animals that had finished OX treatment 3 weeks before (fig. 1).

Effect of $O X$ and CS on passive avoidance and social learning.

In the passive avoidance test, OX rats showed a second latency significantly lower than the control rats (fig. 2); in the social learning test, the time spent by the adult rat in investigating a familiar juvenile rat was not significantly different from the time spent investigating an unfamiliar rat (fig. 3). When CS was co-administered with OX, passive avoidance and social recognition behaviour were not significantly different from controls (figs 2 and 3). Social learning and passive avoidance appeared both impaired in rats that had finished OX treatment 3 weeks before (fig. 4).

\section{Olfactory tests.}

The time spent in locating a preferred food hidden in bedding by rats treated with $\mathrm{OX}$ in the presence/absence of CS or previously submitted to intracranial surgery was not different from that spent by control animals (fig. 5A). The same treatments did not modify the amount of time spent investigating a lemon-scented cylinder during repeated 1-min. presentations, as compared to the values obtained in the control rats (fig. 5B). All rats showed dishabituation when vanilla was added to lemon inside the cylinder (fig. 5B).

\section{Effect of treatment on rat motor behaviour.}

The endurance time on the rotating rod evaluated before and after OX treatment in the presence or absence of CS indicated a lack of any impairment in the motor co-ordination of the treated rats; spontaneous motility as well as inspection activity was unmodified by treatments (fig. 6).

\section{Histology.}

Of the rats that received intracranial injections, three were excluded from statistical analysis because of misplacement. Five rats were excluded for necrosis. Inspection of the remaining brain sections revealed that dye injections were centred within the CA1 hippocampal region (fig. 7).

\section{Vehicle}
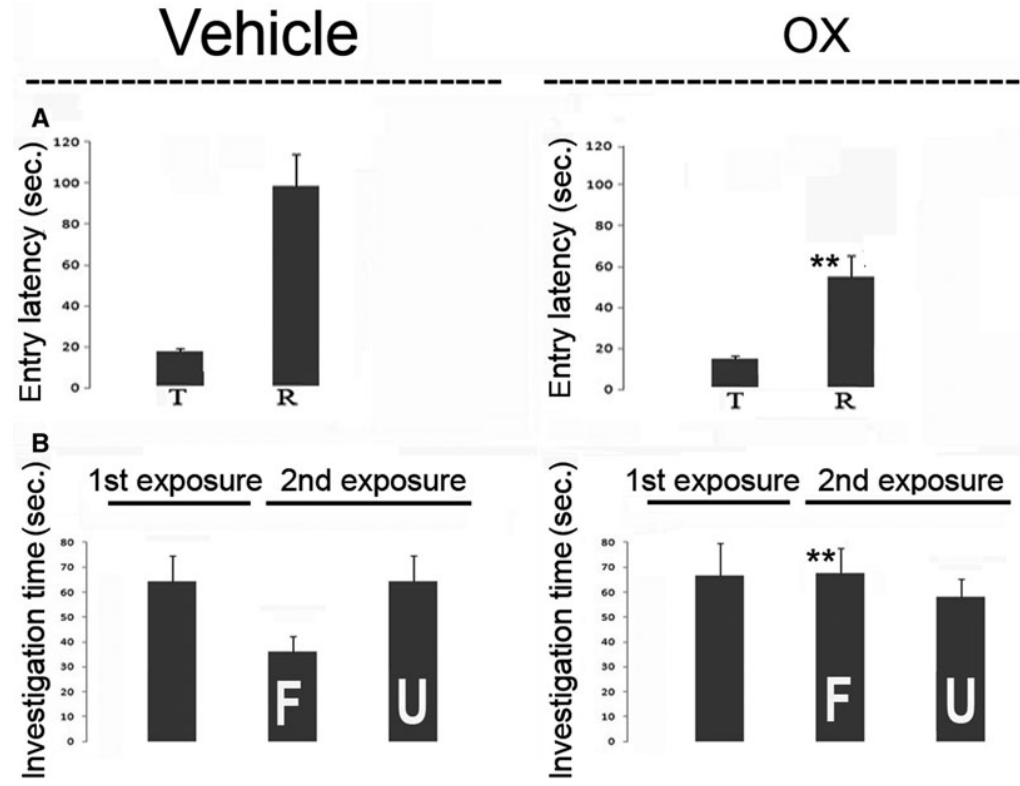

Fig. 4. Result of passive avoidance (A) and social memory test (B) performed in rats 3 weeks after the end of OX treatment. Vertical lines represent S.E.M. Ten rats were used for each experimental condition. ${ }^{* *} \alpha<0.01$ in comparison with corresponding vehicle control. 

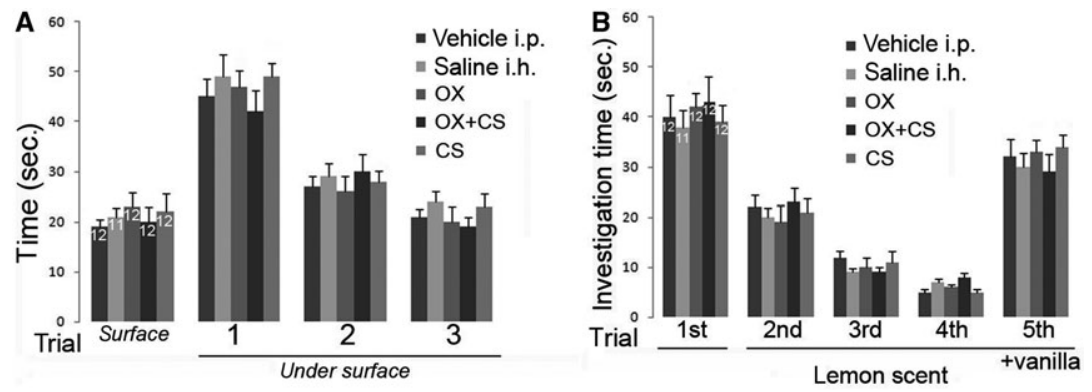

Fig. 5. Olfactory-guided behaviour in rat measured at the end of OX treatment in the presence or absence of CS. (A) Time required by rats to find small pieces of chocolate on the surface or beneath smoothed wood-chip bedding, in three successive trials. (B) Amount of time spent investigating a lemon-scented cylinder during repeated 1-min. presentation training time in the presence of lemon alone or lemon/vanilla combination. Vertical lines represent S.E.M. The number reported on the top of the bar graph represents the number of animals used in each experimental condition. A: $F_{4,54}=1.41$, n.s.; B: $F_{4,54}=1.21$, n.s.
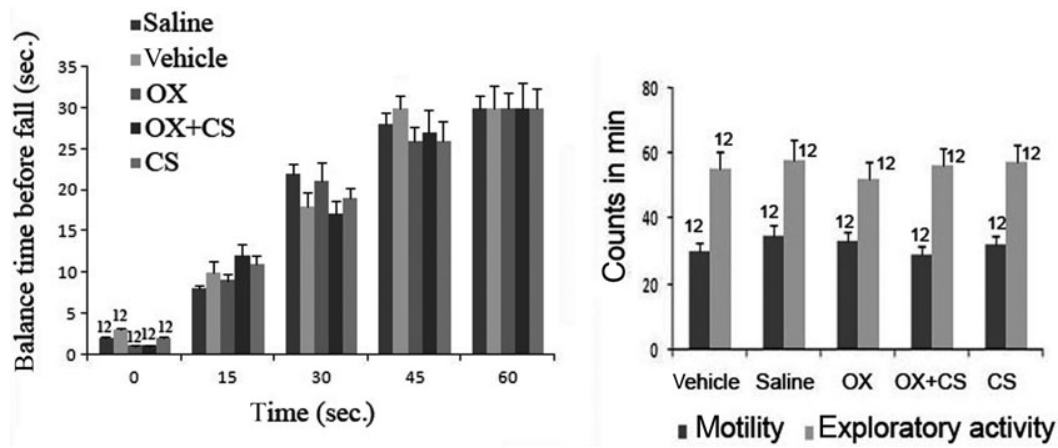

Fig. 6. Lack of effect by OX, OX $+\mathrm{CS}, \mathrm{CS}$, vehicle and saline controls on motor co-ordination in the rotarod test (left). Lack of effect by OX, $\mathrm{OX}+\mathrm{CS}$, CS, vehicle and saline controls on spontaneous motility and exploratory activity (right) in the hole-board test. The tests were performed at the end of OX treatment in the presence or absence of CS. Vertical lines represent S.E.M. The number of animals used in each experimental condition is represented in the figure. Motor co-ordination: $F_{4,54}=1.10$, n.s. Motility: $F_{4,54}=1.72$, n.s. Exploratory activity: $F_{4,54}=1.98$, n.s.

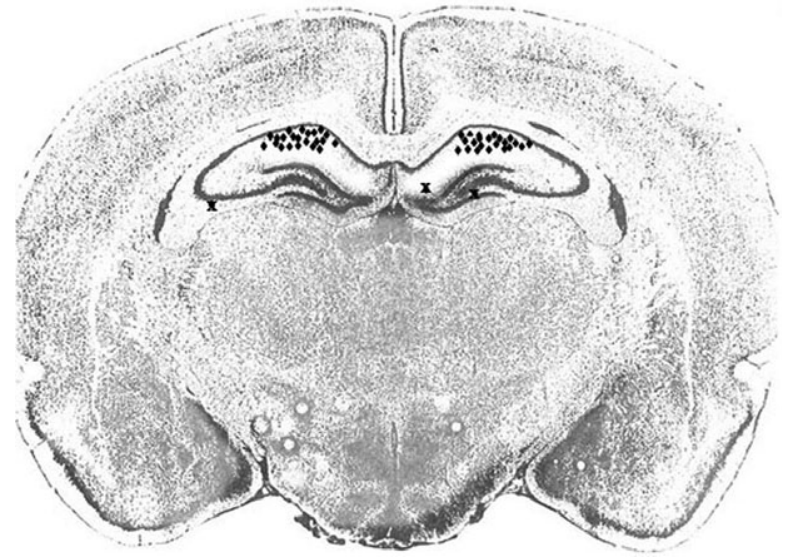

Fig. 7. Schematic representation of microinfusion site distribution within ( ) and outside (x) CA1 hippocampal area of brain of rat. The number of the symbols is less than the total number of rats because of overlaps.

\section{Cultured cell apoptosis.}

The amount of mono- and oligonucleosomes in the cytoplasmic fraction of cell lysates was quantitatively assessed (fig. 8).

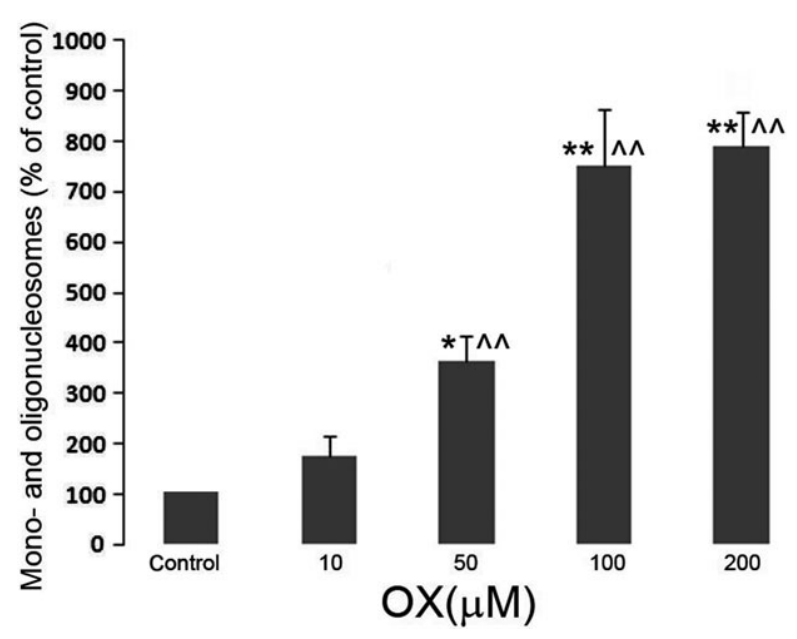

Fig. 8. The amount of cytoplasmic mono- and oligonucleosomes is represented in hippocampal cells incubated with increasing concentrations of OX for $48 \mathrm{hr}$. Each bar represents per cent of control and is obtained from three independent experiments. Reported S.E.M. were obtained from untransformed data. ${ }^{*} \alpha<0.05$ and ${ }^{*} \alpha<<0.01$ in comparison with untreated cells. $F_{4,10}=7.13, \alpha<0.001$. 


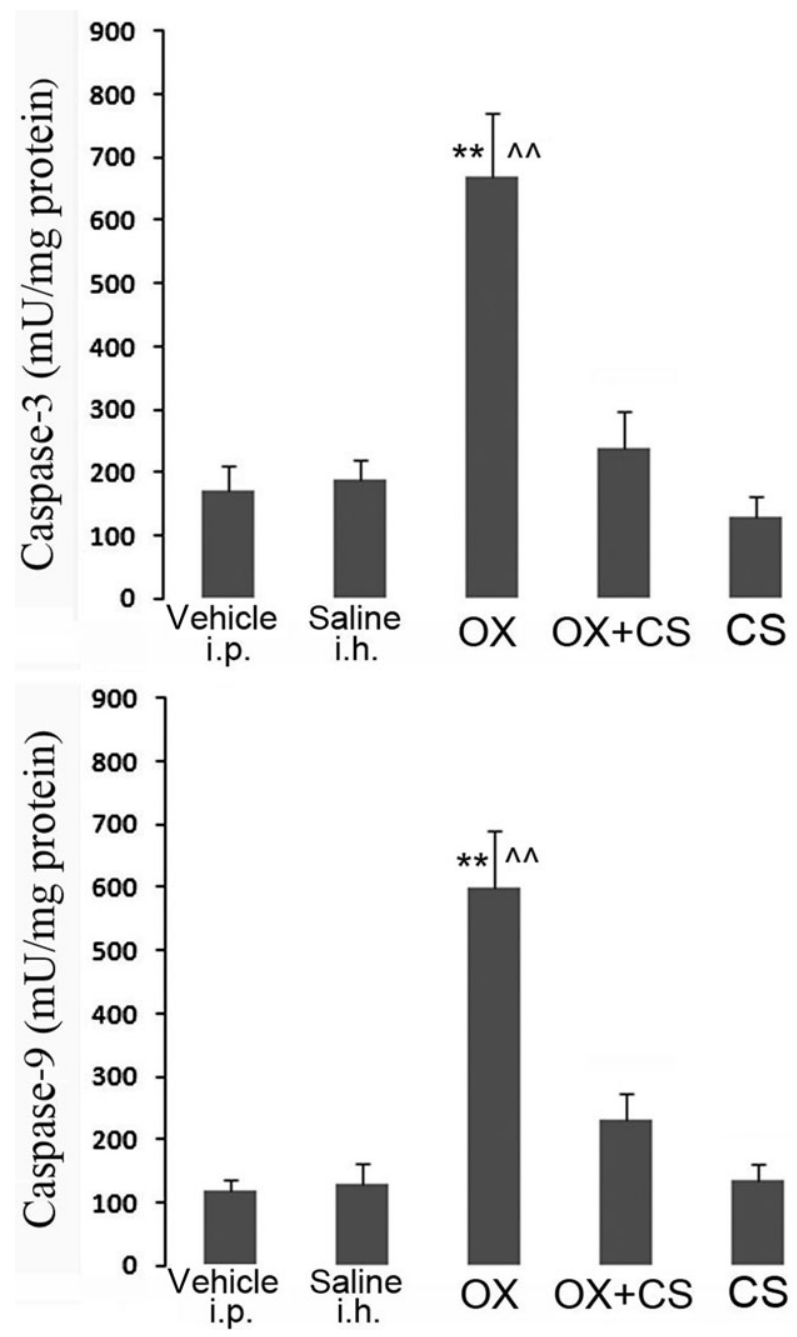

Fig. 9. Effect of 2-week OX treatment in the presence or absence of CS on caspase- 3 and caspase- 9 activity in rat isolated hippocampal tissue. Each bar represents the mean \pm S.E.M. of measured caspase activity and is obtained from four independent experiments, each one made from the tissue of three rats. ${ }^{* *} \alpha<0.01$ and ${ }^{\wedge} \alpha \alpha<0.01$ in comparison with corresponding vehicle and saline controls, respectively. Caspase-3: $F_{4,10}=12.07, \alpha<0.001$. Caspase-9: $F_{4,10}=16.87$, $\alpha<0.001$.

Control culture showed little DNA fragmentation, whereas cells previously treated with $\mathrm{OX}$ for $48 \mathrm{hr}$ showed significantly more mono- and oligonucleosomes at higher OX doses (fig. 8).

\section{Apoptosis marker ex vivo.}

The levels of caspase-3, caspase- 9 and Bax apoptotic proteins significantly increased in the hippocampus from the OX-treated rats as compared to controls (figs 9 and 10). Intracranial co-administration of CS with OX could prevent apoptotic protein increase (figs 9 and 10). In the OX group, Bcl-2 levels were significantly lower than those of the control group, whereas they returned to control values in the presence of CS (fig. 10). OX induced a mitochondrial derangement measured

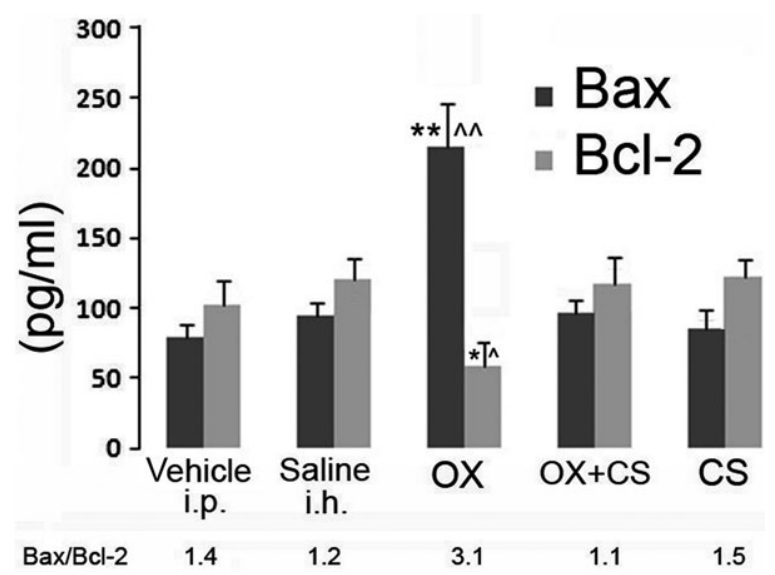

Fig. 10. Change in Bax and Bcl-2 content in hippocampus tissue from rats which underwent 2-week OX treatment. Each bar represents the mean \pm S.E.M. of measured Bax and Bcl-2 concentration in cytosolic fraction. Bax/Bcl-2 ratio is reported in the lower part of the figure. ${ }^{*} \alpha<0.05, *^{*} \alpha<0.01, \wedge^{\wedge} \alpha<0.05, \wedge \wedge \alpha<0.01$, in comparison with vehicle and saline controls. Bax: $F_{4,10}=14.19, \alpha<0.001$. Bcl-2: $F_{4,10}=7.19, \alpha<0.01$

as cytosolic release of cytochrome $\mathrm{c}$ which was reversed by CS administration (fig. 11).

\section{Discussion}

Our experiments have confirmed that repeated treatment with OX induces a cognitive deficit in rat. Lack of any significant decrement in the investigation time taken by the adult rat between the first and second exposure to the juvenile rat demonstrated that social memory formation appeared to be impaired when adult rat was previously subjected to 2-week treatment with OX. It is well known that olfactory system plays a main role in processing olfactory signals relevant to social discrimination [22]. Investigatory behaviour examined in social learning test included direct contact or sniffing, nosing or generally inspecting any body surface of the novel juvenile rat. We were not able to detect any OX-induced alteration in olfactory system of the rats subjected to treatment with the anticancer drug, and therefore, we can exclude that olfactory system impairment has affected the test. Investigation of passive avoidance paradigm revealed that animals previously subjected to 2-week OX treatment developed difficulty in consolidating memory of an unpleasant experience linked to a dark space environment which rodents preferentially explore due to their innate preference of obscurity. Control animals that learned the task avoided the location paired with the aversive stimulus when the possibility of exploring it was offered for the second time. Otherwise, rats previously treated with OX were not able to remember the unpleasant experience associated with the dark space, and the entry latency into this was significantly shorter compared to untreated rats. We observed that OX treatment does not induce any alteration of the motor activity and does not interfere with the exploratory activity of the rats excluding any misinterpretation of results due to these factors. 


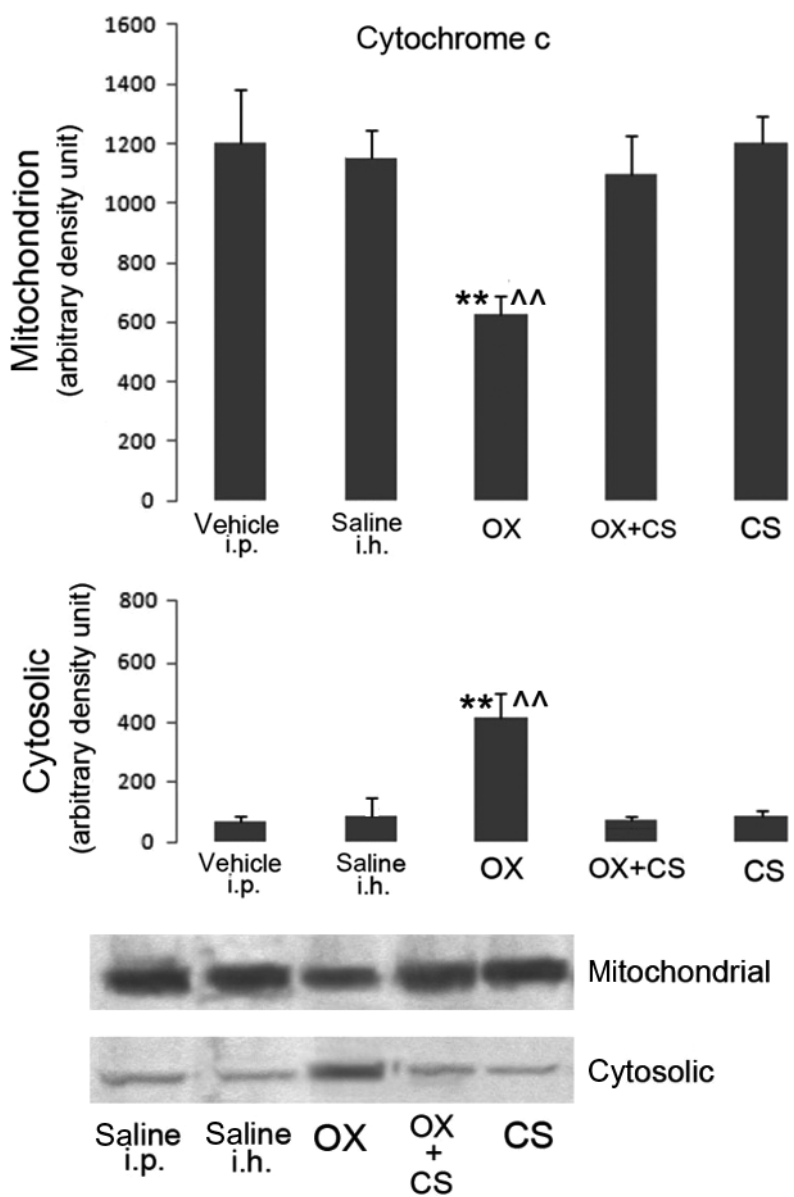

Fig. 11. Cytochrome c in cytosolic and mitochondrial fraction of the hippocampus from rats treated for 2 weeks with OX in the presence or absence of CS. Each bar represents the mean \pm S.E.M. of three independent experiments, each one obtained from four pooled rats. A representative blot is reported in the lower part of the figure. ${ }^{*} \alpha<0.05,{ }^{* *} \alpha<0.01, \wedge^{\wedge} \alpha<0.05, \wedge \wedge \alpha<0.01$, in comparison with vehicle and saline controls. Mitochondrial: $F_{4,15}=6.29, \alpha<0.001$. B: $F_{4,15}=11.89, \alpha<0.001$.

Previous observations that OX has well-described neurotoxic effects [23], penetrates the blood-brain barrier and accumulates in the CNS [5] make conceivable that the drug might have a direct effect on brain function. Hippocampus has been demonstrated as the centre of learning and memory in the CNS $[24,25]$. The ability to make passive avoidance responses was impaired in animals with ablation of the hippocampus [10]. This area plays also a main role in memory for odours on which social learning paradigm is based. Particularly, field CA1 in the hippocampus has been shown to send axons to the main olfactory bulb, the anterior olfactory nucleus and the primary olfactory cortex [26]. The OX-induced memory impairment revealed by our experiments is consistent with hippocampal damage, and therefore, we considered it as an appropriate area for studying the mechanisms involved in the cognitive aspects investigated by our study. In experiments in vitro, apoptosis rate dose-dependently increased in cultured hippocampal cells exposed to OX, as compared to untreated cells. These results were confirmed ex vivo in hippocampal tissue from rats exposed to 2-week OX treatment. We followed, as a guideline for exploring the apoptotic process, OX-induced apoptotic pathway previously shown in colon cancer cells [27]. Activity of main initiator of apoptosis as caspase- 3 and caspase- 9 was significantly increased in the hippocampus from OX-treated rats. A decreased expression of the anti-apoptotic protein Bcl-2 could be observed, which corresponds to a significant increased expression of Bax, a pro-apoptotic protein which, following an apoptotic stimuli, translocates from cytoplasm to mitochondrial membranes mediating the release of cytochrome c. As expected, cytochrome c release could be observed in hippocampal cytosolic fraction from the OX-treated rats. Apoptotic processes were not activated in the hippocampus of rats treated with OX plus CS, a blocker of OX entrance within cells through inhibition of CTR1, a channel-like transporter, localized to the neuronal surface, previously identified as a carrier for OX into the cell $[28,29]$. When CS was delivered locally at both hippocampal sites through bilateral [30] intracranial injections performed concurrently with OX systemic administration, rat hippocampal tissue showed proapoptotic caspase expression levels comparable with those of controls, and cytochrome c content in mitochondrial cell fraction was not decreased. Bax and $\mathrm{Bcl}-2$ relative amount was unmodified with regard to controls. Social memory and passive avoidance paradigms were explored in vivo when OX was prevented to be taken up by hippocampal cells. After 2-week OX treatment in the presence of CS, memory could be consolidated during spontaneous exploration of a novel environment in passive avoidance test, and the knowledge of a new individual could be acquired in social learning behaviour.

Taking our data as a whole, our findings show that a site of action of OX on the two forms of memory explored in this study is located at the hippocampus. At this supraspinal site, cellular damage induced by $\mathrm{OX}$ in this brain area could underlie the weakening of some memory functions in rat.

\section{Conflicts of Interest}

The authors have nothing to disclose.

\section{References}

1 Cruzado JA, López-Santiago S, Martínez-Marín V, José-Moreno G, Custodio AB, Feliu J. Longitudinal study of cognitive dysfunctions induced by adjuvant chemotherapy in colon cancer patients. Support Care Cancer 2014;22:1815-23.

2 Shilling V, Jenkins V. Self-reported cognitive problems in women receiving adjuvant therapy for breast cancer. Eur J Oncol Nurs 2007;11:6-15.

3 Vardy J, Tannock I. Cognitive function after chemotherapy in adults with solid tumours. Crit Rev Oncol Hematol 2007;63:183202.

4 Graham J, Mushin M, Kirkpatrick P. Oxaliplatin. Nat Rev Drug Discovery 2004;3:11-2.

5 Jacobs S, McCully CL, Murphy RF, Bacher J, Balis FM, Fox E. Extracellular fluid concentrations of cisplatin, carboplatin, and oxaliplatin in brain, muscle, and blood measured using microdialysis in nonhuman primates. Cancer Chemother Pharmacol 2010;65:817-24.

6 Fardell JE, Vardy J, Monds LA, Johnston IN. The long-term impact of oxaliplatin chemotherapy on rodent cognition and peripheral neuropathy. Behav Brain Res 2015;291:80-8. 
7 Izquierdo I, Medina JH. Memory formation: the sequence of biochemical events in the hippocampus and its connection to activity in other brain structures. Neurobiol Learn Mem 1997;6:285-316.

8 Kogan JH, Frankland PW, Silva AJ. Long-term memory underlying hippocampus-dependent social recognition in mice. Hippocampus 2000;10:47-56.

9 Izquierdo I, McGaugh JL. Behavioural pharmacology and its contribution to the molecular basis of memory consolidation. Behav Pharmacol 2000;11:517-34.

10 Isaacson RL, Wickelgren WO. Hippocampal ablation and passive avoidance. Science 1962;138:1104-6.

11 Kilkenny C, Browne W, Cuthill IC, Emerson M, Altman DG; NC3Rs Reporting Guidelines Working Group. Br J Pharmacol 2010;160:1577-9.

12 Feinstein AR. Sample size and the other side of statistical significance. In: Clinical Biostatistics. C.V. Mosby Company, St. Louis, 1977; 320-35.

13 Cavaletti G, Tredici G, Petruccioli MG, Dondè E, Tredici P, Marmiroli $\mathrm{P}$ et al. Effects of different schedules of oxaliplatin treatment on the peripheral nervous system of the rat. Eur J Cancer 2001;37:457-63.

14 Kumar V, Kalita J, Misra UK, Bora HK. A study of dose response and organ susceptibility of copper toxicity in a rat model. J Trace Elem Med Biol 2015;29:269-74.

15 Paxinos G, Watson C. The Rat Brain in Stereotaxis Coordinates. Academic Press, New York, 1982.

16 Jarvik ME, Kopp R. An improved one-trial passive avoidance learning situation. Psychol Rep 1967;21:221-4.

17 Dantzer R, Bluthe RM, Koob GF, Le Moal M. Modulation of social memory in male rats by neurohypophyseal peptides. Psychopharmacology 1987;91:363-8.

18 Mencio-Wszalek T, Ramirez VD, Dluzen DE. Age-dependent changes in olfactory-mediated behavioral investigations in the male rat. Behav Neural Biol 1992;57:205-12.

19 Paolini AG, McKenzie JS. Effects of lesions in the horizontal diagonal band nucleus on olfactory habituation in the rat. Neuroscience 1993;57:717-24.

20 Ghelardini C, Galeotti N, Calvani M, Mosconi L, Nicolai R, Bartolini A. Acetyl-1-carnitine induces muscarinic antinociception in mice and rats. Neuropharmacology 2002;43:1180-7.
21 Bianchi E, Norcini M, Smrcka A, Ghelardini C. Supraspinal Gbetagamma-dependent stimulation of PLCbeta originating from G inhibitory protein-mu opioid receptor-coupling is necessary for morphine induced acute hyperalgesia. J Neurochem 2009;111:17180 .

22 Tobin VA, Hashimoto H, Wacker DW, Takayanagi Y, Langnaese $\mathrm{K}$, Caquineau $\mathrm{C}$ et al. An intrinsic vasopressin system in the olfactory bulb is involved in social recognition. Nature 2010;464:413 7.

23 Alcindor T, Beauger N. Oxaliplatin: a review in the era of molecularly targeted therapy. Curr Oncol 2011;18:18-25.

24 Izquierdo LA, Barros DM, Vianna MR, Coitinho A, deDavid e Silva T, Choi $\mathrm{H}$ et al. Molecular pharmacological dissection of short- and long-term memory. Cell Mol Neurobiol 2002;22:26987.

25 Singh KP, Tripathi N. Prenatal exposure to a novel antipsychotic quetiapine: impact on neuro-architecture, apoptotic neurodegeneration in fetal hippocampus and cognitive impairment in young rats. Int J Dev Neurosci 2015;42:59-67.

26 Van Groen T, Wyss JM. Extrinsic projections from area CA1 of the rat hippocampus: olfactory, cortical, subcortical, and bilateral hippocampal formation projections. J Comp Neurol 1990;302:51528.

27 Zanardelli M, Micheli L, Nicolai R, Failli P, Ghelardini C, Di Cesare Mannelli L. Different apoptotic pathways activated by oxaliplatin in primary astrocytes vs. colo-rectal cancer cells. Int J Mol Sci 2015;16:5386-99.

28 Liu JJ, Kim Y, Yan F, Ding Q, Ip V, Jong NN et al. Contributions of rat Ctr1 to the uptake and toxicity of copper and platinum anticancer drugs in dorsal root ganglion neurons. Biochem Pharmacol 2013;85:207-15.

29 Holzer AK, Manorek GH, Howell SB. Contribution of the major copper influx transporter CTR1 to the cellular accumulation of cisplatin, carboplatin, and oxaliplatin. Mol Pharmacol 2006;70: $1390-4$.

30 Di Gennaro G, Grammaldo LG, Quarato PP, Esposito V, Mascia A, Sparano A et al. Severe amnesia following bilateral medial temporal lobe damage occurring on two distinct occasions. Neurol Sci 2006;27:129-33. 